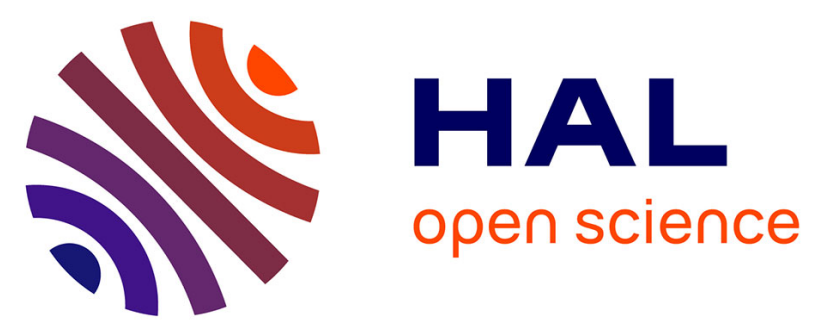

\title{
Impact of alloy disorder on Auger recombination in single InGaN/GaN core-shell microrods
}

W. Liu, G. Rossbach, A. Avramescu, T. Schimpke, H.-J. Lugauer, M. Strassburg, C. Mounir, U. Schwarz, B. Deveaud, Gwenolé Jacopin

\section{- To cite this version:}

W. Liu, G. Rossbach, A. Avramescu, T. Schimpke, H.-J. Lugauer, et al.. Impact of alloy disorder on Auger recombination in single InGaN/GaN core-shell microrods. Physical Review B, 2019, 100 (23), pp.235301. 10.1103/PhysRevB.100.235301 . hal-02397516

\section{HAL Id: hal-02397516 https://hal.science/hal-02397516}

Submitted on 18 Dec 2020

HAL is a multi-disciplinary open access archive for the deposit and dissemination of scientific research documents, whether they are published or not. The documents may come from teaching and research institutions in France or abroad, or from public or private research centers.
L'archive ouverte pluridisciplinaire HAL, est destinée au dépôt et à la diffusion de documents scientifiques de niveau recherche, publiés ou non, émanant des établissements d'enseignement et de recherche français ou étrangers, des laboratoires publics ou privés. 


\title{
Impact of alloy disorder on Auger recombination in single InGaN/GaN core-shell microrods
}

\author{
W. Liu,${ }^{1, *}$ G. Rossbach, ${ }^{2}$ A. Avramescu, ${ }^{2}$ T. Schimpke, ${ }^{2}$ H.-J. Lugauer, ${ }^{2}$ M. \\ Strassburg, ${ }^{2}$ C. Mounir, ${ }^{3}$ U. Schwarz,${ }^{4}$ B. Deveaud, ${ }^{1,5}$ and G. Jacopin ${ }^{1,6}$ \\ ${ }^{1}$ Institute of Physics, École polytechnique fédérale de Lausanne (EPFL), CH-1015 Lausanne, Switzerland \\ ${ }^{2}$ OSRAM Opto Semiconductors GmbH, 93055 Regensburg, Germany \\ ${ }^{3}$ Department of Microsystems Engineering (IMTEK), \\ University of Freiburg, 79110 Freiburg, Germany \\ ${ }^{4}$ Institute of Physics, Technische Universität Chemnitz, 09126 Chemnitz, Germany \\ ${ }^{5}$ École polytechnique, 91128 Palaiseau, France \\ ${ }^{6}$ Institut Néel, Université Grenoble Alpes, CNRS, Grenoble INP, 38000 Grenoble, France
}

(Dated: August 25, 2019)

\begin{abstract}
We study the influence of local inhomogeneities on carrier recombination dynamics in single InGaN/GaN core-shell microrods (MRs) by means of time-resolved micro-photoluminescence (TRPL) at $10 \mathrm{~K}$. At low carrier density $\left(\sim 10^{11} \mathrm{~cm}^{-2}\right)$, the carrier recombination in the $m$-plane quantum well is dominated by radiative processes and the recorded decay times along the MR equally amount to about $400 \mathrm{ps}$, corresponding to a bimolecular coefficient of $1.1 \pm 0.2 \times 10^{-2} \mathrm{~cm}^{2} \mathrm{~s}^{-1}$. When the excited carrier density exceeds $10^{12} \mathrm{~cm}^{-2}$, both the efficiency and the decay time of the PL in the quantum well drop significantly, which indicates the onset of Auger recombination. Based on a modified $A B C$ model, we estimate a $C$ coefficient varying from $0.5 \pm 0.2$ to $2.2 \pm 0.9 \times 10^{-16} \mathrm{~cm}^{4} \mathrm{~s}^{-1}$ from the lower to the upper part of the MR. This increase is accompanied by a rise of PL linewidth in the low excitation regime, indicating an increase of alloy disorder. Relaxation of $k$-selection rule by alloy disorder is expected to play an important role in the observed increase of Auger coefficient. These results confirm that Auger recombination is sensitive to disorder and can be significantly enhanced in strongly disordered systems. We conclude that it is therefore crucial to minimize the degree of disorder in the active layer for high power LEDs based on core-shell MRs.
\end{abstract}

\section{INTRODUCTION}

Thanks to the nonpolar surface orientation, high surface-to-volume ratio, and the absence of non-radiative extended defects, InGaN/GaN core-shell microrods (MRs) have received substantial attention for highly efficient solid state lighting $[1,2]$. They represent one of the potential candidates allowing to mitigate the efficiency droop induced by the increased non-radiative recombination in highly-injected InGaN quantum wells (QWs). Indeed, they allow for a thicker $m$-plane InGaN layer and a significantly larger active region, compared to conventional c-plane QWs [3]. However, core-shell MRs frequently suffer from several intrinsic inhomogeneities, such as a variation of both the In content and the QW thickness $[4,5]$. In particular, because of a limited gas diffusion towards the base of the MRs and due to a higher exposure to the gas-phase precursors near the top of the MR, the selective area growth causes significant inhomogeneities in the sidewall active regions of MRs [6]. Auger recombination is widely accepted as the dominant mechanism underlying the efficiency droop, as proven by the direct observation of linear correlation between hot electron emission current and the droop current [7]. Recent studies further point out that indirect Auger processes may be particularly strong in InGaN/GaN QWs because

\footnotetext{
*we.liu@epfl.ch
}

of the scattering induced by alloy disorder and interface roughness [8-11].

Compared to planar active layers, it is much more demanding to investigate the recombination dynamics in MRs due to their microscopic dimension. A proper analysis of the different contributions to carrier recombination, including Shockley-Read-Hall (SRH), radiative and Auger terms is also essential. Up to date, Auger recombination in single MRs has been studied by confocal pump-probe transmission method and electroluminescence of single MR devices $[12,13]$. However, to our knowledge, the influence of the inhomogeneities on carrier dynamics of Auger processes in single MRs has not been reported yet. Here, relying on a high injection timeresolved photoluminescence setup with $2 \mu \mathrm{m}$ spatial resolution (micro-TRPL) [14], we have explored the carrier dynamics at different positions along a single MR. In combination with a modified $A B C$ model developed previously [11], we are able to extract the excited carrier density, radiative and Auger recombination coefficients, and to evaluate the impact of the different degrees of inhomogeneities on Auger recombination along the MR.

\section{SAMPLE AND MICRO-TRPL SETUPS}

InGaN/GaN core-shell MRs were grown on $n$-GaN/cplane sapphire templates by selective area metalorganic vapour-phase epitaxy [6]. The growth was started with a heavily $n$-doped $\left(\sim 10^{20} \mathrm{~cm}^{-3}\right)$ GaN core grown verti- 
cally [15], under silane-rich condition, which is used to promote the vertical growth [6]. An InGaN underlayer with an $\sim 1 \%$ In-content was grown subsequently, which serves to improve the efficiency of active region $[16,17]$ and prevent the diffusion of background electrons from the core region to the active region. Followed by the shell growth, the active region contains a single InGaN QW in between two GaN barriers. The inner barrier is $n$-type and the outer one is undoped. Each indivual MR has a diameter of $\sim 1.3 \mu \mathrm{m}$ and a length of $\sim 10 \mu \mathrm{m}$. The In content ranges from 11 to $13 \%$ and the width of the InGaN QW increases from 6 to $13 \mathrm{~nm}$ from the bottom to the top of the MR, as characterized by scanning transmission electron microscopy and quantitative analysis of the high-angle annular dark field (HAADF) contrast on MRs grown under similar conditions [5, 18]. More details about the structure can be found in Ref. 19. To investigate individual MRs, a single MR has been detached from the substrate by mechanical lift-off and transferred to a $\mathrm{Si} / \mathrm{SiO}_{2}$ substrate, as the secondary electron image presented in Fig. 1(a).

The experiments were carried out using a micro-TRPL setup. A frequency-tripled Ti:sapphire laser was used as a pump source producing pulses of $280 \mathrm{~nm}$ wavelength and 2 ps duration at a repetition rate of $80.5 \mathrm{MHz}$. The laser beam was focused on the sample by means of a UV objective with $13 \mathrm{~mm}$ focal length and a numerical aperture of 0.32 , allowing to reach a high carrier injection with a photon fluence exceeding $10^{13} \mathrm{~cm}^{-2}$ per pulse. The luminescence spot was magnified 23 times by means of a confocal microscope and refocused on a pinhole of $50 \mu \mathrm{m}$ diameter, which gives a $2.2 \pm 0.2 \mu \mathrm{m}$ spatial resolution. In addition, spatial filtering allowed us to study only the uniform excitation at the center of the laser spot. A streak camera was then used to record carrier dynamics with a temporal resolution better than 20 ps. In order to simplify the analysis and neglect any significant contribution of SRH recombination all measurements in this work were carried out at $10 \mathrm{~K}[19]$.

\section{EMISSION PROPERTIES INVESTIGATED BY MICRO-PL}

To study the influence of the intrinsic inhomogeneities on the local optical properties, we first performed a micro-PL mapping along a single MR at low fluence (0.84 $\mu \mathrm{J} / \mathrm{cm}^{2}$ ) at $10 \mathrm{~K}$. Fig. 1(b) shows the spatial dependence of the time-integrated PL spectra stemming from the $m$-plane QW from the bottom to the top of the MR. Fig. 1(c) shows the corresponding spatially dependent peak energy and full width at half maximum ( $F W H M)$ extracted by Gaussian fitting. Upon moving from the bottom to the top of the MR, we notice a redshift of the QW transition energy from 3.03 down to $2.94 \mathrm{eV}$ together with an increase of the broadening from 140 up to 230 $\mathrm{meV}$. This observation is consistent with the variation of the In content and the QW thickness along the MR as
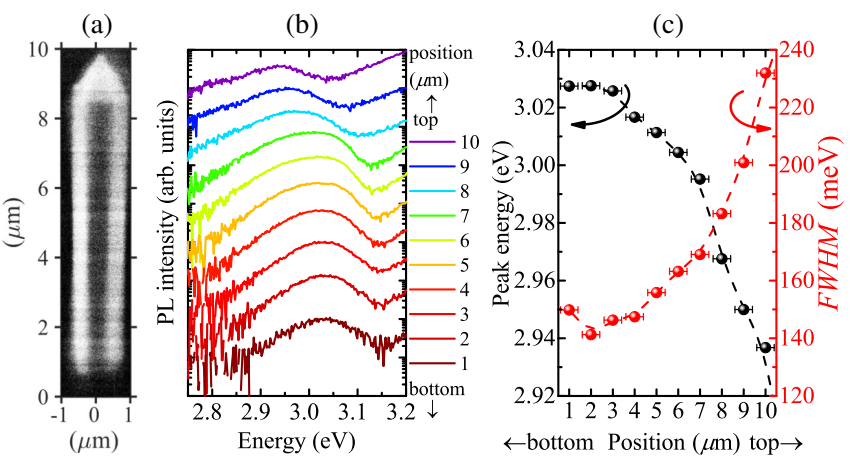

FIG. 1: (a) Plan view secondary electron image of a detached microrod lying on a $\mathrm{Si} / \mathrm{SiO}_{2}$ substrate; (b) One dimensional mapping of the time-integrated PL spectra of the $m$-plane InGaN QW from the bottom to top of the MR under low fluence $0.84 \mu \mathrm{J} / \mathrm{cm}^{2}$ measured at $10 \mathrm{~K}$; (c) the corresponding spatial dependent peak energy (black dots) and full width at half maximum (FWHM) (red dots) obtained by Gaussian fitting of the spectra shown in Fig. 1(b).

specified before. Given the relatively large QW thickness $(>6 \mathrm{~nm})$, the confinement of carriers is predominantly influenced by the local change in the In content. Hence, the variation of the peak energy and the linewidth of the QW along the MR are a result of the In gradient and disorder.

To investigate the Auger-related carrier dynamics in the MR, we then performed fluence dependent microPL and TRPL measurements at $10 \mathrm{~K}$. We probed two positions along the MR with different degrees of disorder, characterized by different peak energy and linewidth. They are used here as an evaluation for the strength of carrier localization [20]. Fig. 2(a) and 2(b) present the fluence dependent time-integrated PL spectra of the $m$ plane QW probed at the lower and upper part of the MR, which correspond to the position at 3 and $8 \mu \mathrm{m}$ shown in Fig. 1, respectively. Fig. 2(c) and 2(d) show the comparison of the QW PL peak energy and the linewidth between the two positions extracted by Gaussian fitting, which illustrate qualitatively the carrier-density dependent blue shift and spectra broadening under different In content and alloy disorder along the MR. In the upper part of the MR, the peak energy of the QW PL clearly blueshifts from 2.94 to $2.97 \mathrm{eV}$ when the fluence rises from 0.21 to $1.68 \mu \mathrm{J} / \mathrm{cm}^{2}$. This goes together with a slight linewidth narrowing from 210 to $180 \mathrm{meV}$. These phenomena are different from the typical narrowing where enhanced scattering between carriers enhances relaxation down to lower localization states, resulting in a redshift of the PL spectrum [21, 22]. Alternatively, this behavior can be explained by the existence of an inplane electric field along the $<0001>$ direction induced by In-rich clusters with a few nm size [5]. In other words, the screening of the field not only results in a blueshift, but also reduces the field-induced potential fluctuations 
(a)
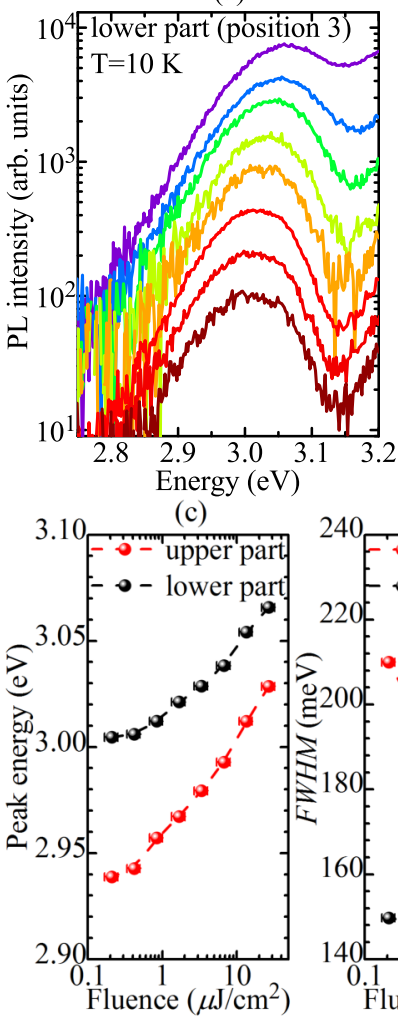

(b)

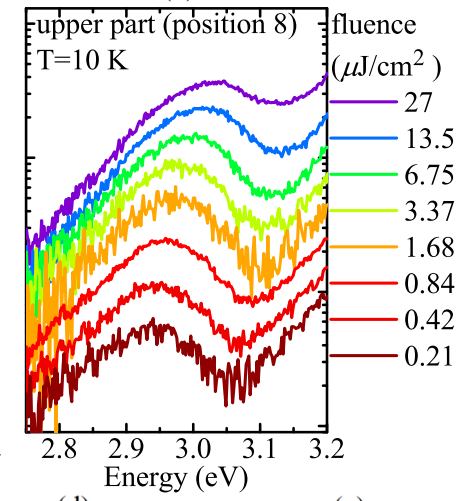

(d)

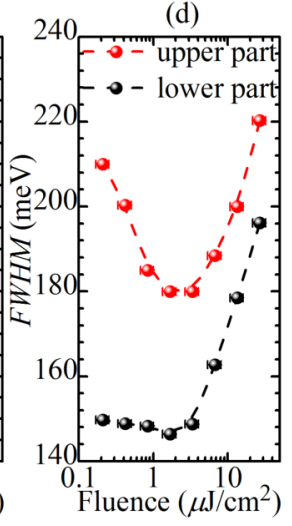

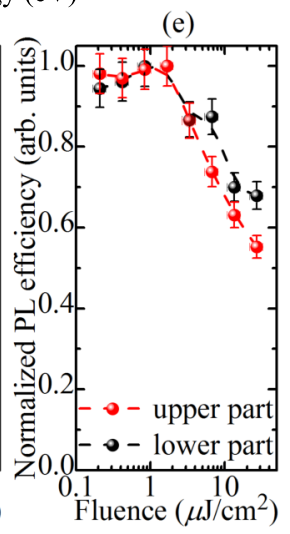

FIG. 2: Fluence dependent time-integrated PL spectra of the $m$-plane $\mathrm{QW}$ recorded in the lower and the upper part of the MR. (a) and (b) correspond to the probing position at 3 and $8 \mu \mathrm{m}$ in Fig. 1, respectively. (c) and (d) the fluence dependent peak energy and FWHM of QW PL measured in the lower (black dots) and in the upper (red dots) part of the MR extracted by Gaussian fitting. (e) Derived PL efficiencies of the QW at the two recorded positions (normalized ratio between PL intensity and its corresponding fluence).

$[23,24]$. For the lower part of the MR, characterized with a lower In content, the blueshift (3.00-3.02 eV) and linewidth narrowing (150-146 meV) are less pronounced, which is in line with our interpretation. In addition, the fluence dependent spectrum evolution can be also related to the interplay between inhomogeneous broadening and the degenerate carrier statistics [25].

As the fluence increases to its maximum $\left(27 \mu \mathrm{J} / \mathrm{cm}^{2}\right)$, a clear blueshift as well as a broadening of the spectra at both positions hints the strong phase-space filling under high injection. The fluence dependent PL efficiency of the InGaN QW emission is then calculated as the ratio between the PL intensity and the corresponding fluence, referenced to the value obtained at low fluence, as shown in Fig. 2(e). For both positions, the efficiency shows a plateau at low injection and drops when the fluence increases up to $27 \mu \mathrm{J} / \mathrm{cm}^{2}$, evidencing the presence of the droop. Moreover, without anticipating the following TRPL results, we can notice that the decrease of the relative efficiency is more pronounced in the upper part of the MR than in the lower one.

\section{CARRIER DYNAMICS PROBED BY MICRO-TRPL AND MODELING}

To gain more insight into carrier dynamics of the InGaN QW under high injection, we performed timeresolved studies. Fig. 3 (a) and 3 (b) show the fluence dependent PL decays integrated over the full energy range of QW luminescence measured in the lower and in the upper part of the MR, respectively. At low fluence, below
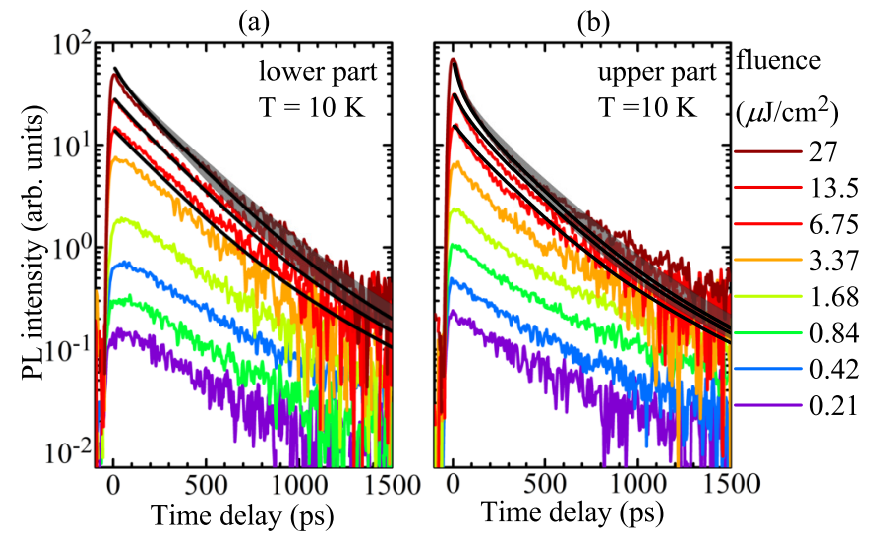

FIG. 3: (a) and (b) fluence dependent PL decays (color lines) of the $m$-plane QW measured in the lower and in the upper parts of the MR, and corresponding best fittings (black lines), and the tolerant ones (grey shaded zones) for the decay at $27 \mu \mathrm{J} / \mathrm{cm}^{2}$ based on a modified $A B C$ model.

$0.84 \mu \mathrm{J} / \mathrm{cm}^{2}$, the two positions share a similar and invariant single exponential decay, which is consistent with the observed plateau of PL efficiency in Fig. 2(e) and suggests a dominant radiative recombination process. When the fluence goes beyond $6.75 \mu \mathrm{J} / \mathrm{cm}^{2}$, both series of decays exhibit a fast non-exponential trend at early delay. Note that, under high injection, phase-space filling results in a constant radiative lifetime evidenced by the linear increase of PL intensity at early delays [26-28]. Additionally, even if SRH recombination was present, its saturation at high carrier density would contribute to a longer decay, not a shorter one [11,28]. Consequently, the observed non-exponential decay strongly indicates the emergence of Auger recombination.

Fig. 4(a) and 4(b) illustrate the time-dependent evolutions of the spectra of the $m$-plane QW at the two recorded positions. At early delay $(t=0)$, the spectra at both positions reveal a pronounced broadening of the QW PL induced by the high carrier density. At longer decay, both series of spectra show a noticeable redshift, which can be attributed to the carrier relaxation from shallow localization states to deeper ones and to the descreening of the in-plane field [29]. Moreover, it is appar- 

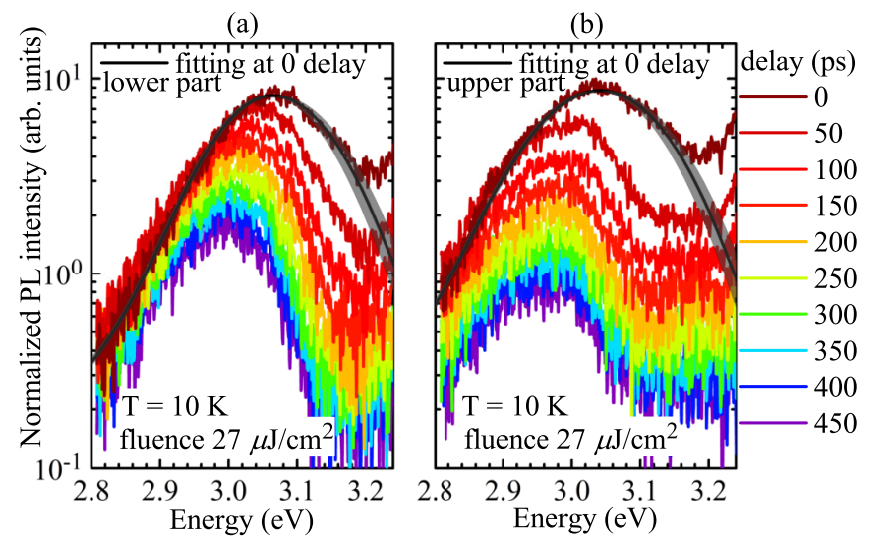

FIG. 4: (a) and (b) time-dependent InGaN spectra evolution (from 0 to $450 \mathrm{ps}$ ) of the $m$-plane QW measured in the lower and in the upper part of the MR under high injection $\left(27 \mu \mathrm{J} / \mathrm{cm}^{2}\right)$ at $10 \mathrm{~K}$, and corresponding tolerant (grey shaded zones) and best (black lines) fittings of the spectra at early delay $(t=0)$ based on Fermi's golden rule.

ent that the time-dependent PL intensity for the upper part of the MR drops much faster than that in the lower part, which suggests a stronger Auger recombination in the former case.

In order to quantify the coefficient of Auger recombination from TRPL results, we use a modified $A B C$ model $[11,30]$ :

$$
\frac{d n}{d t}=-\int_{E_{Q W}}^{\infty} R_{s p}(n, E) d E-\frac{C}{2}\left(\left(n+n_{0}\right)^{2} n+\left(n+n_{0}\right) n^{2}\right)
$$

where $n, n_{0}$, and $C$ denote the non-equilibrium carrier density, the electron background concentration, and the Auger recombination coefficient, respectively. $R_{s p}$ is the spontaneous radiative rate, which can be expressed as:

$$
R_{s p}(n, E)=\sum_{j=A, B} \frac{1}{\tau_{0}} D(E)_{j}^{c v} f(n, E)_{e, j} f(n, E)_{h, j}
$$

where $\tau_{0}$ is the average transition lifetime, which depends on the oscillator strength, $D(E)$ is the 2D joint density of states. $f_{e, j}, f_{h, j}$ are the electron and hole Fermi-Dirac distribution, with the subscript indicating the transitions from the conduction to the $\mathrm{A}$ and $\mathrm{B}$ valence bands. The consideration of those two transitions is referred to the report that they are the dominant components measured in polarization-resolved PL spectra when carrier density reaches the degenerate regime [25]. In the last term of Eq. 1, the prefactor $1 / 2$ is used to allow for a comparison with the standard $A B C$ model by assuming an equal contribution between electron-electron-hole and electronhole-hole Auger processes [8]. SRH recombination is neglected as mentioned before. The radiative lifetime $\tau_{r}$, Auger lifetime $\tau_{A u g}$, and the effective lifetime $\tau_{\text {eff }}$ at early delay can then be obtained via:

$$
\begin{gathered}
\tau_{r}=\frac{n}{\int_{E_{Q W}}^{\infty} R_{s p}(n, E) d E}, \\
\tau_{\text {Aug }}=\frac{2}{C\left[\left(n+n_{0}\right)^{2}+\left(n+n_{0}\right) n\right]}, \\
\tau_{\text {eff }}=\left(\tau_{r}^{-1}+\tau_{\text {Aug }}^{-1}\right)^{-1} .
\end{gathered}
$$

The fitting parameters include $n$ at $t=0, n_{0}, \tau_{0}$ and $C$, which are determined as follows. First, the initial $n$ at $27 \mu \mathrm{J} / \mathrm{cm}^{2}$ is extracted through a fit of the spectral shape at $t=0$, using Eq. 2 convoluted with a Gaussian profile reproducing the inhomogeneous linewidth [11, 29]. The linewidth is taken at a moderate excitation flux of 3.37 $\mu \mathrm{J} / \mathrm{cm}^{2}$, corresponding to the minimum value in Fig. 2 (d). The results of such best fits and tolerant fits dependent on the uncertainties of $n$ are displayed in Fig. 4. At low excitation density values, the corresponding initial $n$ at each fluence can be derived assuming a linearity between the laser fluence and the initial carrier density. Then, $\tau_{0}, n_{0}$ and $C$, summarized in Table I, serve to fit simultaneously the PL decay and the initial PL intensity $I_{p l}(t=0)$ in Fig. 3, and the experimental effective lifetimes $\tau_{\text {eff }}$ in Fig. 5. Specifically, $\tau_{\text {eff }}$ denotes the effective carrier lifetime for each fluence and is obtained by fitting the initial decay by a single exponential. The modeling reveals that the $C$ coefficient shows a variation by about a factor of 4 between the upper and the lower part of the MR with values of $2.2 \pm 0.9$ and $0.5 \pm 0.2 \times 10^{-16} \mathrm{~cm}^{4} \mathrm{~s}^{-1}$, respectively. The estimated value in $3 \mathrm{D}$ units amounts to $2.7 \pm 2.0 \times 10^{-28}$ and $1.8 \pm 1.0 \times 10^{-29} \mathrm{~cm}^{6} \mathrm{~s}^{-1}$, respectively, by using $\sim 11$ and $6 \mathrm{~nm}$ QW thickness at the two positions of the MR [5]. On the other hand, the $B$ coefficient can be estimated by $1 /\left[\left(n_{0}+n\right) \tau_{r}\right]$ and has similar values at both positions, around $1.1 \pm 0.2 \times 10^{-2} \mathrm{~cm}^{2} \mathrm{~s}^{-1}$ at low carrier density $\left(\sim 10^{11} \mathrm{~cm}^{-2}\right)$, which corresponds to $1.21 \pm 0.44$ and $0.66 \pm 0.18 \times 10^{-8} \mathrm{~cm}^{3} \mathrm{~s}^{-1}$ in $3 \mathrm{D}$ units. These extracted coefficients are consistent with our previous report about planar $m$-plane QW with strong alloy disorder at $4 \mathrm{~K}[11]$.

Finally, Fig. 5 compares the changes of $\tau_{\text {eff }}$ of the InGaN QW in the lower and in the upper parts of the $\mathrm{MR}$ as a function of the injected carrier density. At low carrier density $\left(\sim 10^{11} \mathrm{~cm}^{-2}\right)$, $\tau_{\text {eff }}$ shows similar values for both positions ( $\sim 400 \mathrm{ps})$. The slightly longer $\tau_{\text {eff }}$ observed at the top of the MR can be ascribed to the lower electron background concentration $n_{0}$ as listed in Table I [15]. By contrast, at maximum carrier density, $\tau_{\text {eff }}$ varies significantly between the upper and the lower part of the rod, with values of 70 and 160 ps, respectively. In addition, the $\tau_{\text {eff }}$ of the barrier exhibited in Fig. 5 is as short as $\sim 100 \mathrm{ps,} \mathrm{which} \mathrm{suggests} \mathrm{an} \mathrm{efficient}$ carrier transfer to the InGaN QW and no dependence on the actual position along the MR. These observations 
TABLE I: Summaries of predominant parameters for fitting and the experimental linewidths

\begin{tabular}{lccccc}
\hline \hline position & $n\left(\times 10^{12} \mathrm{~cm}^{-2}\right)$ & $\tau_{0}\left(\times 10^{-6} \mathrm{~s}\right)$ & $C\left(\times 10^{-16} \mathrm{~cm}^{4} \mathrm{~s}^{-1}\right)$ & $F W H M(\mathrm{meV})$ & $n_{0}\left(\times 10^{11} \mathrm{~cm}^{-2}\right)$ \\
\hline upper & $9.5 \pm 1.0$ & $5.1 \pm 0.5$ & $2.2 \mp 0.9$ & $180 \pm 10$ & $1.0 \pm 0.3$ \\
lower & $8.5 \pm 1.0$ & $5.0 \pm 0.5$ & $0.5 \mp 0.2$ & $148 \pm 10$ & $1.2 \pm 0.3$ \\
\hline \hline
\end{tabular}

(a)

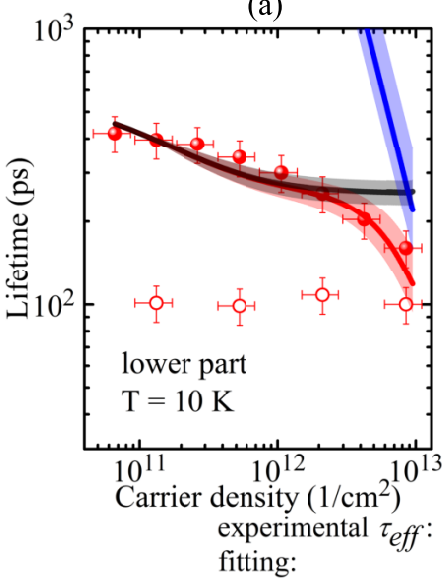

(b)

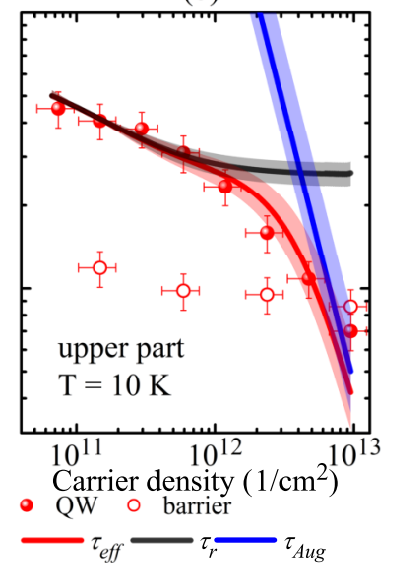

FIG. 5: (a) and (b) experimental effective PL lifetime $\tau_{\text {eff }}$ in the InGaN QW (solid dots) and the GaN barrier (hollow circles) as a function of carrier density measured in the lower and in the upper part of the MR, extracted from a single exponential decay in Fig. 3, and corresponding tolerant (shaded zones) and best (solid lines) theoretical estimations of $\tau_{\text {eff }}$ (red), radiative lifetime $\tau_{r}$ (black) and Auger one $\tau_{\text {Aug }}$ (blue).

lead us to the conclusion that the decrease of $\tau_{\text {eff }}$ under high injection is mainly governed by Auger recombination. Moreover, based on the distinct drop in PL efficiency together with the shorter $\tau_{\text {eff }}$ in the InGaN QW closer to the top of the MR, the stronger Auger recombination is likely related to the inhomogeneous linewidth and thus to the disorder landscape. These observations are in agreement with the outcome of previous studies on planar $m$-plane samples [8-11]. Using Eqs. 3 and 4 enables us to distinguish between the contribution of $\tau_{r}$ and $\tau_{A u g}$ to the carrier recombination dynamics, as shown in Fig. 5. As the QW carrier density increases from $10^{11}$ to $10^{12} \mathrm{~cm}^{-2}, \tau_{r}$ governs the evolution of $\tau_{e f f}$, and decreases continuously from $\sim 400$ to $\sim 300$ ps. When $n$ is near $10^{13} \mathrm{~cm}^{-2}, \tau_{r}$ is expected to be constant and equal to $\sim 260 \mathrm{ps}$, due to phase-space filling [26-28], and the corresponding internal quantum efficiency in the lower and upper part of MR is about $62 \%$ and $27 \%$, respectively, given by $\tau_{\text {eff }} / \tau_{r}$. Meanwhile, $\tau_{\text {Aug }}$ dramatically drops and become shorter than $\tau_{r}$ when $n$ is approaching to $10^{13} \mathrm{~cm}^{-2}$. Interestingly, we observe that the $\tau_{A u g}$ is 4 times shorter under high injection in the upper part of the MR compared to the lower one. Therefore, considering the similar $\tau_{r}$ value in the two positions, it suggests that stronger Auger recombination takes place in

the upper part. This can be understood by taking into account the stronger alloy disorder in this region as characterized by the larger PL linewidth and In content. In order to elucidate the underlying mechanism, we qualitatively model the relaxation of the $k$-selection rule in presence of alloy disorder and its impact on Auger recombination, as detailed in the supplementary. Based on localization landscape theory and Schrödinger equation $[31,32]$, our simulation reveals that the ground state carriers are localized within one of the potential minima with a few nanometer spatial extension. As In content is increasing, the spatial extension of ground state is further reduced, which corresponds to a broadening in $k$-space. Finally, we model [10] the dependence of three-particle Auger process on the finite linewidth. It indicates that a low $k$-broadening (diluted In content) restrains the direct Auger transition due to the stringent $k$-selection rule. In contrast, as In content increases, the Auger coefficient increases significantly, which results from the relaxation of $k$-selection rule by the strong alloy disorder.

\section{CONCLUSION}

In conclusion, by using micro-TRPL at $10 \mathrm{~K}$, we study the local carrier recombination dynamics in a single dispersed core-shell MR with an $m$-plane InGaN/GaN QW. The impact of InGaN alloy disorder on the carrier dynamics is quantified by a modified $A B C$ model. At low carrier density, the carrier recombination is dominated by radiative processes and the recorded decay times along the MR equally amount to about $400 \mathrm{ps}$, corresponding to a $B$ coefficient of $1.1 \pm 0.2 \times 10^{-2} \mathrm{~cm}^{2} \mathrm{~s}^{-1}$. As a comparison, at high carrier density $\left(>10^{12} \mathrm{~cm}^{-2}\right)$, the efficiency and decay time of PL in the QW drop significantly, which indicates the onset of Auger recombination. The extracted $C$ coefficient near the top part of the MR is about 4 times larger than that at the base, which is manifested by values of $2.2 \pm 0.9$ and $0.5 \pm 0.2 \times 10^{-16} \mathrm{~cm}^{4} \mathrm{~s}^{-1}$, respectively. This is related to a different degree of alloy disorder in the two probed positions, with 180 and 150 $\mathrm{meV}$ inhomogeneous linewidth, respectively. Relaxation of $k$-selection rule by ally disorder is expected to play an important role in the increase of Auger coefficient. Therefore, our results confirm that Auger recombination is sensitive to alloy disorder and can be significantly enhanced when this type of disorder is prominent. Our results suggest that it is crucial to minimize the degree of disorder in the active layer for high power light-emitting diodes based on core-shell MRs. 


\section{ACKNOWLEDGMENTS}

This work was financially supported by the Swiss Na- tional Science Foundation under Project No. 154853. The authors acknowledge Nicolas Grandjean and Raphaël Butté for their constructive advice on this study.
[1] A. Waag, X. Wang, S. Fündling, J. Ledig, M. Erenburg, R. Neumann, M. Al Suleiman, S. Merzsch, J. Wei, S. Li, et al., physica status solidi (c) 8, 2296 (2011).

[2] S. Li and A. Waag, Journal of Applied Physics 111, 071101 (2012).

[3] C. Weisbuch, M. Piccardo, L. Martinelli, J. Iveland, J. Peretti, and J. S. Speck, physica status solidi (a) 212, 899 (2015).

[4] M. Tchernycheva, P. Lavenus, H. Zhang, A. Babichev, G. Jacopin, M. Shahmohammadi, F. Julien, R. Ciechonski, G. Vescovi, and O. Kryliouk, Nano Letters 14, 2456 (2014).

[5] M. Müller, P. Veit, F. F. Krause, T. Schimpke, S. Metzner, F. Bertram, T. Mehrtens, K. Müller-Caspary, A. Avramescu, M. Strassburg, et al., Nano letters 16, 5340 (2016).

[6] M. Mandl, X. Wang, T. Schimpke, C. Kölper, M. Binder, J. Ledig, A. Waag, X. Kong, A. Trampert, F. Bertram, et al., physica status solidi (RRL)-Rapid Research Letters 7, 800 (2013).

[7] J. Iveland, L. Martinelli, J. Peretti, J. S. Speck, and C. Weisbuch, Physical Review Letters 110, 177406 (2013).

[8] E. Kioupakis, D. Steiauf, P. Rinke, K. T. Delaney, and C. G. Van de Walle, Physical Review B 92, 035207 (2015).

[9] C. Jones, C.-H. Teng, Q. Yan, P.-C. Ku, and E. Kioupakis, Applied Physics Letters 111, 113501 (2017).

[10] C.-K. Tan, W. Sun, J. J. Wierer Jr, and N. Tansu, AIP Advances 7, 035212 (2017).

[11] M. Shahmohammadi, W. Liu, G. Rossbach, L. Lahourcade, A. Dussaigne, C. Bougerol, R. Butté, N. Grandjean, B. Deveaud, and G. Jacopin, Physical Review B 95, 125314 (2017).

[12] S. Boubanga-Tombet, J. B. Wright, P. Lu, M. R. Williams, C. Li, G. T. Wang, and R. P. Prasankumar, ACS Photonics 3, 2237 (2016).

[13] Y. Hou, J. Bai, R. Smith, and T. Wang, Nanotechnology 27, 205205 (2016).

[14] M. Shahmohammadi, G. Jacopin, G. Rossbach, J. Levrat, E. Feltin, J.-F. Carlin, J.-D. Ganiere, R. Butté, N. Grandjean, and B. Deveaud, Nature Communications 5, 5251 (2014).

[15] M. Mohajerani, S. Khachadorian, T. Schimpke, C. Nenstiel, J. Hartmann, J. Ledig, A. Avramescu, M. Strassburg, A. Hoffmann, and A. Waag, Applied Physics Letters 108, $091112(2016)$.
[16] A. M. Armstrong, B. N. Bryant, M. H. Crawford, D. D. Koleske, S. R. Lee, and J. J. Wierer Jr, Journal of Applied Physics 117, 134501 (2015).

[17] C. Haller, J.-F. Carlin, G. Jacopin, W. Liu, D. Martin, R. Butté, and N. Grandjean, Applied Physics Letters 113, 111106 (2018).

[18] C. Mounir, T. Schimpke, G. Rossbach, A. Avramescu, M. Strassburg, and U. T. Schwarz, Journal of Applied Physics 120, 155702 (2016).

[19] W. Liu, C. Mounir, G. Rossbach, T. Schimpke, A. Avramescu, H.-J. Lugauer, M. Strassburg, U. Schwarz, B. Deveaud, and G. Jacopin, Applied Physics Letters 112, 052106 (2018).

[20] M. Piccardo, C.-K. Li, Y.-R. Wu, J. S. Speck, B. Bonef, R. M. Farrell, M. Filoche, L. Martinelli, J. Peretti, and C. Weisbuch, Physical Review B 95, 144205 (2017).

[21] R. Zimmermann, F. Grosse, and E. Runge, Pure and Applied Chemistry 69, 1179 (1997).

[22] M. Vallone, M. Goano, F. Bertazzi, and G. Ghione, Journal of Applied Physics 121, 123107 (2017).

[23] F. Natali, D. Byrne, M. Leroux, B. Damilano, F. Semond, A. Le Louarn, S. Vezian, N. Grandjean, and J. Massies, Physical Review B 71, 075311 (2005).

[24] H. Wang, Z. Ji, S. Qu, G. Wang, Y. Jiang, B. Liu, X. Xu, and H. Mino, Optics Express 20, 3932 (2012).

[25] L. Schade, T. Wernicke, J. Rass, S. Ploch, M. Weyers, M. Kneissl, and U. Schwarz, physica status solidi (b) 253, 145 (2016).

[26] A. McAllister, D. Bayerl, and E. Kioupakis, Applied Physics Letters 112, 251108 (2018).

[27] E. Kioupakis, Q. Yan, D. Steiauf, and C. G. Van de Walle, New Journal of Physics 15, 125006 (2013).

[28] W. Liu, R. Butté, A. Dussaigne, N. Grandjean, B. Deveaud, and G. Jacopin, Physical Review B 94, 195411 (2016).

[29] M. Shahmohammadi, J.-D. Ganière, H. Zhang, R. Ciechonski, G. Vescovi, O. Kryliouk, M. Tchernycheva, and G. Jacopin, Nano letters 16, 243 (2016)

[30] G. Rossbach, J. Levrat, G. Jacopin, M. Shahmohammadi, J.-F. Carlin, J.-D. Ganière, R. Butté, B. Deveaud, and N. Grandjean, Physical Review B 90, 201308(R) (2014).

[31] D. N. Arnold, G. David, D. Jerison, S. Mayboroda, and M. Filoche, Physical review letters 116, 056602 (2016).

[32] M. Filoche, M. Piccardo, Y.-R. Wu, C.-K. Li, C. Weisbuch, and S. Mayboroda, Physical Review B 95, 144204 (2017). 